\title{
Fixed-Point Theorems for $\alpha$-Admissible Mappings with $w$-Distance and Applications to Nonlinear Integral Equations
}

\author{
Fengrong Zhang, ${ }^{1}$ Haoyue Wang, ${ }^{2}$ Shuangqi $W u,{ }^{3}$ and Liangshi Zhao ${ }^{4}{ }^{4}$ \\ ${ }^{1}$ School of Information Science and Engineering, Dalian Polytechnic University, Dalian, Liaoning 116034, China \\ ${ }^{2}$ Department of Mathematics, Liaoning Normal University, Dalian, Liaoning 116029, China \\ ${ }^{3}$ Huludao No. 6 Senior High School, Huludao, Liaoning 125000, China \\ ${ }^{4}$ Center for Studies of Marine Economy and Sustainable Development, Liaoning Normal University, Dalian \\ Liaoning 116029, China
}

Correspondence should be addressed to Liangshi Zhao; liangshizhao85@163.com

Received 30 November 2019; Accepted 26 February 2020; Published 28 March 2020

Academic Editor: Rosana Rodríguez López

Copyright ( $) 2020$ Fengrong Zhang et al. This is an open access article distributed under the Creative Commons Attribution License, which permits unrestricted use, distribution, and reproduction in any medium, provided the original work is properly cited.

Two fixed-point theorems for $\alpha$-admissible mappings satisfying contractive inequality of integral type with $w$-distance in complete metric spaces are proved. Our results extend and improve a few existing results in the literature. As applications, we use the fixedpoint theorems obtained in this paper to establish solvability of nonlinear integral equations. Examples are included.

\section{Introduction and Preliminaries}

In 2002, Branciari [1] discussed the existence and uniqueness of fixed points for mappings satisfying contractive condition of integral type, which is a generalization of the Banach contraction principle in metric spaces.

Theorem 1 (see [1]). Let $f$ be a mapping from a complete metric space $(X, d)$ into itself satisfying

$$
\int_{0}^{\mathrm{d}(f x, f y)} \phi(t) \mathrm{d} t \leq c \int_{0}^{\mathrm{d}(x, y)} \phi(t) \mathrm{d} t,
$$

for all $x, y \in X$, where $c \in(0,1)$ is a constant and $\phi \in \Phi_{2}$. Then, $f$ has a unique fixed point $a \in X$ and $\lim _{n \longrightarrow \infty} f^{n} x=a$ for each $x \in X$.

In 2012, Samet et al. [2] introduced the concept of $\alpha-\psi$-contractive type mappings and established some fixedpoint theorems for these mappings in complete metric spaces.

Definition 1 (see [2]). Let $(X, d)$ be a metric space, $T: X \longrightarrow X$ and $\alpha: X \times X \longrightarrow \mathbb{R}^{+}$be two given mappings. Then, $T$ is called an $\alpha$-admissible mapping if

$$
\alpha(x, y) \geq 1 \Rightarrow \alpha(T x, T y) \geq 1, \quad \forall x, y \in X
$$

Theorem 2 (see [2]). Let $(X, d)$ be a complete metric space and $T: X \longrightarrow X$ be an $\alpha-\psi$-contractive mapping, that is,

$$
\alpha(x, y) d(T x, T y) \leq \psi(d(x, y)), \quad \forall x, y \in X,
$$

where $\psi \in \Phi_{1}$. Assume that

$$
\begin{aligned}
& \left(a_{1}\right) T \text { is } \alpha \text {-admissible } \\
& \left(a_{2}\right) \text { there exists } x_{0} \in X \text { such that } \alpha\left(x_{0}, T x_{0}\right) \geq 1 \\
& \left(a_{3}\right) T \text { is continuous }
\end{aligned}
$$

Then, $T$ has a fixed point, that is, there exists $x^{*} \in X$ such that $T x^{*}=x^{*}$.

Theorem 3 (see [2]). Let $(X, d)$ be a complete metric space and $T: X \longrightarrow X$ satisfy (3), $\left(a_{1}\right),\left(a_{2}\right)$, and the following

$\left(a_{4}\right)$ if $\left\{x_{n}\right\}_{n \in \mathbb{N}}$ is a sequence in $X$ such that $\alpha\left(x_{n}, x_{n+1}\right) \geq 1$ for all $n \in \mathbb{N}$ and $x_{n} \longrightarrow x \in X$ as $n \longrightarrow \infty$, then $\alpha\left(x_{n}, x\right) \geq 1$ for all $n \in \mathbb{N}$.

Then, $T$ has a fixed point. 
In 1996, Kada et al. [3] introduced the notion of $w$-distance and elaborated with the help of examples, that the concept of $w$-distance is general than that of metric on a nonempty set. Several researchers [4-7] used this notion to obtain some fixed point results not only in usual metric spaces but also in other spaces.

Definition 2 (see [3]). Let $(X, d)$ be a metric space. A function $p: X \times X \longrightarrow \mathbb{R}^{+}$is called a $w$-distance in $X$ if it satisfies the following:

$\left(p_{1}\right) p(x, z) \leq p(x, y)+p(y, z), \forall x, y, z \in X$

$\left(p_{2}\right)$ for each $x \in X$, a mapping $p(x, \cdot): X \longrightarrow \mathbb{R}^{+}$is lower semicontinuous

$\left(p_{3}\right)$ for any $\varepsilon>0$, there exists $\delta>0$ such that $p(z, x) \leq \delta$ and $p(z, y) \leq \delta$ imply $d(x, y) \leq \varepsilon$.

In 2016, Lakzian et al. [8] introduced the concept of generalized $(\alpha-\psi-p)$-contractive mappings and proved the following fixed point results for such mappings, which generalize Theorems 2 and 3.

Theorem 4 (see [8]). Let $p$ be a $w$-distance on a complete metric space $(X, d)$, and let $f: X \longrightarrow X$ be an $(\alpha-\psi-$ p)-contractive mapping, that is,

$$
\alpha(x, y) p(f x, f y) \leq \psi(p(x, y)), \quad \forall x, y \in X,
$$

where $\psi \in \Phi_{1}$. Assume that $\left(b_{1}\right) f$ is an $\alpha$-admissible mapping

$\left(b_{2}\right)$ there exists a point $x_{0} \in X$ such that $\alpha\left(x_{0}, f x_{0}\right) \geq 1$

$\left(b_{3}\right)$ either $f$ is continuous or, for any sequence $\left\{x_{n}\right\}_{n \in \mathbb{N}}$ in $X$ if $\alpha\left(x_{n}, x_{n+1}\right) \geq 1$ for all $n \in \mathbb{N}$ and $x_{n} \longrightarrow x \in X$ as $n \longrightarrow \infty$, then $\alpha\left(x_{n}, x\right) \geq 1$ for all $n \in \mathbb{N}$.

Then, there exists a point $u \in X$ such that $f u=u$. Moreover, if $\alpha(u, u) \geq 1$, then $p(u, u)=0$.

Theorem 5 (see [8]). Let $p$ be a $w$-distance on a complete metric space $(X, d)$, and let $f: X \longrightarrow X$ satisfy (4), ( $\left.b_{1}\right)$, $\left(b_{2}\right)$, and the following

$\left(b_{4}\right)$ for every $y \in X$ with $y \neq f y$, inf $\{p(x, y)+p(x$, $f x): x \in X\}>0$.

Then, there exists a point $u \in X$ such that $f u=u$. Moreover, if $\alpha(u, u) \geq 1$, then $p(u, u)=0$.

Motivated by the results in [1-10], in this paper we prove the existence and uniqueness of fixed points for $\alpha$-admissible mappings satisfying contractive inequality of integral type via $w$-distance in complete metric spaces, which are used to study solvability of nonlinear Fredholm and Volterra integral equations. Our results generalize Theorems 1-5 and two examples are given.

Throughout this paper, we denote by $\mathbb{N}$ the set of positive integers, $\mathbb{N}_{0}=\{0\} \cup \mathbb{N}, \mathbb{R}=(-\infty,+\infty), \mathbb{R}^{+}=[0,+\infty)$, and

$$
\begin{aligned}
& \Phi_{1}=\left\{\psi \mid \psi: \mathbb{R}^{+} \longrightarrow \mathbb{R}^{+} \text {satisfies that } \psi \text { is nondecreasing and } \sum_{n=1}^{\infty} \psi^{n}(t)<+\infty, \forall t>0\right\} \\
& \Phi_{2}=\left\{\phi \mid \phi: \mathbb{R}^{+} \longrightarrow \mathbb{R}^{+} \text {is Lebesgue integrable, summable in each compact subset of } \mathbb{R}^{+} \text {and } \int_{0}^{\varepsilon} \phi(t) \mathrm{d} t>0, \forall \varepsilon>0\right\} \\
& \Phi_{3}=\left\{\phi \mid \phi \text { is in } \Phi_{2} \text { and } \int_{0}^{a+b} \phi(t) \mathrm{d} t \leq \int_{0}^{a} \phi(t) \mathrm{d} t+\int_{0}^{b} \phi(t) \mathrm{d} t, \forall a, b \in \mathbb{R}^{+}\right\}
\end{aligned}
$$

Lemma 1 (see [10]). Let $\phi \in \Phi_{2}$ and $\left\{r_{n}\right\}_{n \in \mathbb{N}}$ be a nonnegative sequence. Then, $\lim _{n \longrightarrow \infty} \int_{0}^{r_{n}^{2}} \phi(t) d t=0$ if and only if $\lim _{n \rightarrow \infty} r_{n}=0$.

\section{Fixed-Point Theorems}

In this section, we prove the existence and uniqueness of fixed points for $\alpha$-admissible mapping (6) with $w$-distance.

Theorem 6. Let $p$ be a $w$-distance in a complete metric space $(X, d)$ and let $f: X \longrightarrow X$ satisfy that

$$
\alpha(x, y) \int_{0}^{p(f x, f y)} \phi(t) \mathrm{d} t \leq \psi\left(\int_{0}^{p(x, y)} \phi(t) \mathrm{d} t\right), \quad \forall x, y \in X,
$$

where $(\psi, \phi) \in \Phi_{1} \times \Phi_{3}$. Assume that

$\left(c_{1}\right) f$ is an $\alpha$-admissible mapping

$\left(c_{2}\right)$ there exists a point $x_{0} \in X$ such that $\alpha\left(x_{0}, f x_{0}\right) \geq 1$ if one of the following conditions holds:

$\left(c_{3}\right) f$ is continuous

$\left(c_{4}\right)$ for any sequence $\left\{x_{n}\right\}_{n \in \mathbb{N}}$ in $X$ if $\alpha\left(x_{n}, x_{n+1}\right) \geq 1$ for all $n \in \mathbb{N}$ and $x_{n} \longrightarrow x \in X$ as $n \longrightarrow \infty$, then $\alpha\left(x_{n}, x\right) \geq 1$ for all $n \in \mathbb{N}$ Then, (1) $f$ has a fixed point $u \in X$ and $\lim _{n \longrightarrow \infty} f^{n} x_{0}=u$. Moreover, $p(u, u)=0$ if $\alpha(u, u) \geq 1$.

(2) fhas a unique fixed point $u \in X$ if for any fixed points $x, y \in X$ of $f$, there exists a point $z \in X$ such that $\alpha(z, x) \geq 1$ and $\alpha(z, y) \geq 1$.

Proof. Firstly, we show (1). Define a sequence $\left\{x_{n}\right\}_{n \in \mathbb{N}}$ in $X$ by $x_{n+1}=f x_{n}, \forall n \in \mathbb{N}_{0}$, where $x_{0}$ satisfies $\left(c_{2}\right)$. Assume that there exists some $n_{0} \in \mathbb{N}$ with $x_{n_{0}}=x_{n_{0}-1}$. Put $u=x_{n_{0}-1}$. Clearly, $u$ is a fixed point of $f$ and $u=\lim _{n \longrightarrow \infty} f^{n} x_{0}$.

Now, we assume that $x_{n} \neq x_{n-1}$ for all $n \in \mathbb{N}$. It follows from $\left(c_{1}\right)$ and $\left(c_{2}\right)$ that 
$\alpha\left(x_{0}, x_{1}\right)=\alpha\left(x_{0}, f x_{0}\right) \geq 1 \Rightarrow \alpha\left(x_{1}, x_{2}\right)=\alpha\left(f x_{0}, f x_{1}\right) \geq 1$.

It is easy to see that

$$
\alpha\left(x_{n}, x_{n+1}\right) \geq 1, \quad \forall n \in \mathbb{N}_{0} .
$$

In view of (6) and (8) and $(\psi, \phi) \in \Phi_{1} \times \Phi_{3}$, we conclude that

$$
\begin{aligned}
0 & \leq \int_{0}^{p\left(x_{n}, x_{n+1}\right)} \phi(t) \mathrm{d} t=\int_{0}^{p\left(f x_{n-1}, f x_{n}\right)} \phi(t) \mathrm{d} t \\
& \leq \alpha\left(x_{n-1}, x_{n}\right) \int_{0}^{p\left(f x_{n-1}, f x_{n}\right)} \phi(t) \mathrm{d} t \leq \psi\left(\int_{0}^{p\left(x_{n-1}, x_{n}\right)} \phi(t) \mathrm{d} t\right) \\
& \leq \psi^{n}\left(\int_{0}^{p\left(x_{0}, x_{1}\right)} \phi(t) \mathrm{d} t\right) \longrightarrow 0 \text { as } n \longrightarrow \infty,
\end{aligned}
$$

which implies that

$$
\lim _{n \longrightarrow \infty} \int_{0}^{p\left(x_{n}, x_{n+1}\right)} \phi(t) \mathrm{d} t=0,
$$

which together with $(\psi, \phi) \in \Phi_{1} \times \Phi_{3}$ and Lemma 1 yields that

$$
\lim _{n \longrightarrow \infty} p\left(x_{n}, x_{n+1}\right)=0 .
$$

Let $\varepsilon>0$ and $\delta$ be defined by $\left(p_{3}\right)$. Note that $\sum_{n=1}^{\infty} \psi^{n}\left(\int_{0}^{p\left(x_{0}, x_{1}\right)} \phi(t) \mathrm{d} t\right)<+\infty$, which means that there exists $n_{0} \in \mathbb{N}$ satisfying

$$
\sum_{k=n_{0}}^{\infty} \psi^{k}\left(\int_{0}^{p\left(x_{0}, x_{1}\right)} \phi(t) \mathrm{d} t\right)<\int_{0}^{\delta} \phi(t) \mathrm{d} t .
$$

In the following, we claim that $\left\{x_{n}\right\}_{n \in \mathbb{N}}$ is a Cauchy sequence. Making use of (6), (9), (12), ( $\left.p_{1}\right)$, and $(\psi, \phi) \in \Phi_{1} \times \Phi_{3}$, we infer that

$$
\begin{aligned}
\int_{0}^{p\left(x_{n}, x_{m}\right)} \phi(t) \mathrm{d} t & \leq \int_{0}^{\sum_{k=n}^{m-1} p\left(x_{k}, x_{k+1}\right)} \phi(t) \mathrm{d} t \leq \sum_{k=n}^{m-1} \int_{0}^{p\left(x_{k}, x_{k+1}\right)} \phi(t) \mathrm{d} t \\
& \leq \sum_{k=n}^{m-1} \psi^{k}\left(\int_{0}^{p\left(x_{0}, x_{1}\right)} \phi(t) \mathrm{d} t\right) \\
& <\int_{0}^{\delta} \phi(t) \mathrm{d} t, \quad \forall m, n \in \mathbb{N} \text { with } m>n \geq n_{0},
\end{aligned}
$$

which implies that

$$
p\left(x_{n}, x_{m}\right)<\delta, \quad \forall m, n \in \mathbb{N} \text { with } m>n \geq n_{0} .
$$

It follows from (14) that

$$
\begin{aligned}
& p\left(x_{n_{0}}, x_{n}\right)<\delta, \\
& p\left(x_{n_{0}}, x_{m}\right)<\delta, \quad \forall m, n \in \mathbb{N} \text { with } m>n \geq n_{0},
\end{aligned}
$$

which together with $\left(p_{3}\right)$ gives that

$$
d\left(x_{n}, x_{m}\right)<\varepsilon, \quad \forall m, n \in \mathbb{N} \text { with } m>n \geq n_{0},
$$

That is, $\left\{x_{n}\right\}_{n \in \mathbb{N}}$ is a Cauchy sequence. By completeness of $X$, there exists a point $u \in X$ such that

$$
\lim _{n \longrightarrow \infty} x_{n}=u \text {. }
$$

Assume that $\left(c_{3}\right)$ holds. Using (17) and $\left(c_{3}\right)$, we gain that

$$
u=\lim _{n \longrightarrow \infty} x_{n+1}=\lim _{n \longrightarrow \infty} f x_{n}=f\left(\lim _{n \longrightarrow \infty} x_{n}\right)=f u \text {. }
$$

Assume that $\left(c_{4}\right)$ holds. By virtue of (8), (17), and $\left(c_{4}\right)$, we deduce that

$$
\alpha\left(x_{n}, u\right) \geq 1, \quad \forall n \in \mathbb{N} .
$$

Similar to the proofs of (12)-(14), we know that for each $\varepsilon_{1}>0$ there exists $n_{1} \in \mathbb{N}$ satisfying

$$
0 \leq p\left(x_{n}, x_{m}\right)<\varepsilon_{1}, \quad \forall m, n \in \mathbb{N} \text { with } m>n \geq n_{1},
$$

which together with $\left(p_{2}\right)$ and (17) yields that

$$
0 \leq p\left(x_{n}, u\right) \leq \liminf _{m \longrightarrow \infty} p\left(x_{n}, x_{m}\right) \leq \varepsilon_{1}, \quad \forall n \in \mathbb{N} \text { with } n \geq n_{1},
$$

that is,

$$
\lim _{n \longrightarrow \infty} p\left(x_{n}, u\right)=0
$$

In light of (6), (19), and $(\psi, \phi) \in \Phi_{1} \times \Phi_{3}$, we obtain that

$$
\begin{aligned}
0 & \leq \int_{0}^{p\left(x_{n+1}, f u\right)} \phi(t) \mathrm{d} t=\int_{0}^{p\left(f x_{n}, f u\right)} \phi(t) \mathrm{d} t \leq \alpha\left(x_{n}, u\right) \int_{0}^{p\left(f x_{n}, f u\right)} \phi(t) \mathrm{d} t \\
& \leq \psi\left(\int_{0}^{p\left(x_{n}, u\right)} \phi(t) \mathrm{d} t\right) \leq \psi^{n+1}\left(\int_{0}^{p\left(x_{0}, u\right)} \phi(t) \mathrm{d} t\right) \longrightarrow 0 \text { as } n \longrightarrow \infty,
\end{aligned}
$$

which together with Lemma 1 implies that

$$
\lim _{n \longrightarrow \infty} p\left(x_{n+1}, f u\right)=0 .
$$

In view of (11), (24) and, $\left(p_{1}\right)$, we infer that

$0 \leq p\left(x_{n}, f u\right) \leq p\left(x_{n}, x_{n+1}\right)+p\left(x_{n+1}, f u\right) \longrightarrow 0$ as $n \longrightarrow \infty$,

that is,

$$
\lim _{n \longrightarrow \infty} p\left(x_{n}, f u\right)=0 .
$$

Let $\varepsilon_{2}>0$. It follows from $\left(p_{3}\right)$ that there exists $\delta_{1}>0$ such that $p(u, v) \leq \delta_{1}$ and $p(u, z) \leq \delta_{1}$ imply $d(v, z)<\varepsilon_{2}$. Combining (22) and (26), we know that there exists $n_{2} \in \mathbb{N}$ such that $p\left(x_{n}, u\right) \leq \delta_{1}$ and $p\left(x_{n}, f u\right) \leq \delta_{1}$ for all $n \geq n_{2}$. Hence, $d(u, f u) \leq \varepsilon_{2}$. Letting $\varepsilon_{2} \longrightarrow 0^{+}$, we have

$$
u=f u \text {. }
$$

Next, we show that $p(u, u)=0$ if $\alpha(u, u) \geq 1$. Suppose that $p(u, u)>0$. In view of $(6)$ and $(\psi, \phi) \in \Phi_{1} \times \Phi_{3}$, we infer that 


$$
\begin{aligned}
0 & <\int_{0}^{p(u, u)} \phi(t) \mathrm{d} t=\int_{0}^{p(f u, f u)} \phi(t) \mathrm{d} t \leq \alpha(u, u) \int_{0}^{p(f u, f u)} \phi(t) \mathrm{d} t \\
& \leq \psi\left(\int_{0}^{p(u, u)} \phi(t) \mathrm{d} t\right)<\int_{0}^{p(u, u)} \phi(t) \mathrm{d} t,
\end{aligned}
$$

which is ridiculous. Hence, $p(u, u)=0$.

Secondly, we show (2). Suppose that $x$ and $y$ are two fixed points of $f$ in $X$. It follows that there exists a point $z \in X$ satisfying

$$
\begin{aligned}
& \alpha(z, x) \geq 1, \\
& \alpha(z, y) \geq 1 .
\end{aligned}
$$

In light of (29) and $\left(c_{1}\right)$, we get that

$$
\begin{aligned}
& \alpha\left(f^{n} z, x\right) \geq 1 \\
& \alpha\left(f^{n} z, y\right) \geq 1, \quad \forall n \in \mathbb{N},
\end{aligned}
$$

which together with (6) and $(\psi, \phi) \in \Phi_{1} \times \Phi_{3}$ imply that

$$
\begin{aligned}
0 & \leq \int_{0}^{p\left(f^{n+1} z, x\right)} \phi(t) \mathrm{d} t=\int_{0}^{p\left(f f^{n} z, f x\right)} \phi(t) \mathrm{d} t \\
& \leq \alpha\left(f^{n} z, x\right) \int_{0}^{p\left(f f^{n} z, f x\right)} \phi(t) \mathrm{d} t \leq \psi\left(\int_{0}^{p\left(f^{n} z, f x\right)} \phi(t) \mathrm{d} t\right) \\
& \leq \psi^{n}\left(\int_{0}^{p(f z, x)} \phi(t) \mathrm{d} t\right) \longrightarrow 0 \text { as } n \longrightarrow \infty,
\end{aligned}
$$

which gives that

$$
\lim _{n \longrightarrow \infty} \int_{0}^{p\left(f^{n+1} z, x\right)} \phi(t) \mathrm{d} t=0 .
$$

Similarly, we conclude that

$$
\lim _{n \longrightarrow \infty} \int_{0}^{p\left(f^{n+1} z, y\right)} \phi(t) \mathrm{d} t=0 .
$$

Making use of (32), (33), and Lemma 1, we get that

$$
\begin{aligned}
& \lim _{n \longrightarrow \infty} p\left(f^{n+1} z, x\right)=0, \\
& \lim _{n \longrightarrow \infty} p\left(f^{n+1} z, y\right)=0,
\end{aligned}
$$

which together with the proof of (27) yields similarly that $x=y$. That is, $f$ has a unique fixed point in $X$. This completes the proof.

Theorem 7. Let $p$ be a $w$-distance in a complete metric space $(X, d)$, and let $f: X \longrightarrow X$ satisfy $(6),\left(c_{1}\right),\left(c_{2}\right)$, and the following

$\left.\left(c_{5}\right) \inf \{p(x, y)+p(x, f x): x \in X\}\right\}>0$ for each $y \in X$ with $y \neq f y$.

Then, (1) and (2) hold.

Proof. Following the proof of Theorem 6, we deduce that $\left\{x_{n}\right\}_{n \in \mathbb{N}}$ is a Cauchy sequence. By completeness of $(X, d)$, there exists a point $u \in X$ that satisfies (17) holds. Suppose that $u \neq f u$. In light of (11), (17), and $\left(c_{5}\right)$, we conclude that

$$
\begin{aligned}
0 & <\inf \{p(x, u)+p(x, f x): x \in X\} \\
& \leq \inf \left\{p\left(x_{n}, u\right)+p\left(x_{n}, x_{n+1}\right): n \in \mathbb{N}\right\} \\
& =0,
\end{aligned}
$$

which is impossible. Hence, $u=f u$. The rest of the proof is similar to that of Theorem 6 and is omitted. This completes the proof.

Remark 1. Theorem 6 generalizes and improves Theorems $1-4$. The following example manifests that Theorem 6 extends substantially Theorem 1 .

Example 1. Let $X=[0,(1 / 2)] \cup\{2 / 3\} \cup\{1\}$ be endowed with the Euclidean metric $d=|\cdot|, \quad p: X \times X \longrightarrow \mathbb{R}^{+}$, $\psi, \phi: \mathbb{R}^{+} \longrightarrow \mathbb{R}^{+}, f: X \longrightarrow X$, and $\alpha: X \times X \longrightarrow \mathbb{R}^{+}$be defined by

$$
\begin{aligned}
p(x, y) & =y, \quad \forall x, y \in X, \\
\psi(t) & =\frac{4}{5} t, \\
\phi(t) & =1, \quad \forall t \in \mathbb{R}^{+},
\end{aligned}
$$

$$
f x= \begin{cases}\frac{2}{3} x^{2}, & \forall x \in\left[0, \frac{1}{2}\right], \\ \frac{1}{2}, & x=\frac{2}{3} \\ \frac{2}{3}, & x=1,\end{cases}
$$

$$
\alpha(x, y)= \begin{cases}1, & \text { if } x, y \in\left[0, \frac{1}{2}\right] \cup\left\{\frac{2}{3}\right\}, \\ 0, & \text { otherwise. }\end{cases}
$$

It is clear that $p$ is a $w$-distance in $X$ and $(\psi, \phi) \in \Phi_{1} \times \Phi_{3}$. Let $x, y \in X$. In order to verify (6), we have to consider the following cases.

Case 1. $x \in[0,(1 / 2)] \cup\{2 / 3\}$ and $y \in[0,(1 / 2)]$. It follows that

$$
\begin{aligned}
\alpha(x, y) \int_{0}^{p(f x, f y)} \phi(t) \mathrm{d} t & =\int_{0}^{(2 / 3) y^{2}} \mathrm{dt}=\frac{2}{3} y^{2} \leq \frac{4}{5} y=\psi(y) \\
& =\psi\left(\int_{0}^{p(x, y)} \phi(t) \mathrm{d} t\right) .
\end{aligned}
$$


Case 2. $x \in[0,(1 / 2)] \cup\{2 / 3\}$ and $y=(2 / 3)$. Note that

$$
\begin{aligned}
\alpha(x, y) \int_{0}^{p(f x, f y)} \phi(t) \mathrm{d} t & =\int_{0}^{1 / 2} \mathrm{~d} t=\frac{1}{2} \leq \frac{4}{5} \cdot \frac{2}{3}=\psi\left(\frac{2}{3}\right) \\
& =\psi\left(\int_{0}^{p(x, y)} \phi(t) \mathrm{d} t\right) .
\end{aligned}
$$

Case 3. $x \notin[0,(1 / 2)] \cup\{2 / 3\}$ or $y \notin[0,(1 / 2)] \cup\{2 / 3\}$. It is clear that

$$
\alpha(x, y) \int_{0}^{p(f x, f y)} \phi(t) \mathrm{d} t=0 \leq \psi\left(\int_{0}^{p(x, y)} \phi(t) \mathrm{d} t\right) .
$$

That is, (6) holds. Let $x, y \in X$ with $\alpha(x, y) \geq 1$. It follows that $x, y \in[0,(1 / 2)] \cup\{2 / 3\}$ and

$$
\begin{aligned}
& f x= \begin{cases}\frac{2}{3} x^{2}, & \forall x \in\left[0, \frac{1}{2}\right], \\
\frac{1}{2}, & x=\frac{2}{3},\end{cases} \\
& f y= \begin{cases}\frac{2}{3} y^{2}, & \forall y \in\left[0, \frac{1}{2}\right], \\
\frac{1}{2}, & y=\frac{2}{3},\end{cases}
\end{aligned}
$$

which imply that $f x \in[0,(1 / 2)]$ and $f y \in[0,(1 / 2)]$, that is, $\alpha(f x, f y)=1$. Hence, $f$ is an $\alpha$-admissible mapping.

Put $x_{0}=(2 / 3) \in X$. It is clear that $\alpha\left(x_{0}, f x_{0}\right)=$ $\alpha((2 / 3),(1 / 2))=1$.

Finally, let $\left\{x_{n}\right\}_{n \in \mathbb{N}}$ be a sequence in $X$ such that $\alpha\left(x_{n}, x_{n+1}\right) \geq 1$ for all $n \in \mathbb{N}$ and $x_{n} \longrightarrow x \in X$ as $n \longrightarrow \infty$. It is easy to see that $x_{n} \in[0,(1 / 2)] \cup\{2 / 3\}$ for all $n \in \mathbb{N}$. Since $\left\{x_{n}\right\}_{n \in \mathbb{N}}$ is a sequence in the closed subset $[0,(1 / 2)] \cup\{2 / 3\}$ in the metric space $(X, d)$, it follows that the point $x$ belongs to $[0,(1 / 2)] \cup\{2 / 3\}$. Therefore, $\alpha\left(x_{n}, x\right)=1$ for all $n \in \mathbb{N}$.

Hence, the conditions of Theorem 6 are satisfied. It follows from Theorem 6 that $f$ has a fixed point $0 \in X$.

However, we cannot invoke Theorem 1 to show that the mapping $f$ has a fixed point in $X$. Suppose that the conditions of Theorem 1 are satisfied. Clearly, $y_{*}=(2 / 3)$.

$$
\begin{aligned}
0 & <\int_{0}^{1 / 3} \phi(t) \mathrm{d} t=\int_{0}^{d(f(1 / 2), f(2 / 3))} \phi(t) \mathrm{d} t \leq c \int_{0}^{d((1 / 2),(2 / 3))} \phi(t) \mathrm{d} t \\
& <\int_{0}^{1 / 6} \phi(t) \mathrm{d} t \leq \int_{0}^{1 / 3} \phi(t) \mathrm{d} t
\end{aligned}
$$

which is impossible. Thus, Theorem 1 is not applicable in proving the existence of fixed points for the mapping $f$ in $X$.

Remark 2. Theorem 7 generalizes Theorem 5. The example below is an application of Theorem 7 .

Example 2. Let $X=\{0\} \cup\left\{\left(1 /(n+1)^{2}\right): n \in \mathbb{N}\right\}$ be endowed with the Euclidean metric $d=|\cdot|, \quad p: X \times X \longrightarrow \mathbb{R}^{+}$, $\psi, \phi: \mathbb{R}^{+} \longrightarrow \mathbb{R}^{+}, f: X \longrightarrow X$, and $\alpha: X \times X \longrightarrow \mathbb{R}^{+}$be defined by

$$
\begin{aligned}
p(x, y) & =y^{2}, \quad \forall x, y \in X, \\
\psi(t) & =\frac{1}{2} t, \\
\phi(t) & =1, \quad \forall t \in \mathbb{R}^{+}, \\
f x & =x^{2}, \quad \forall x \in X \\
\alpha(x, y) & = \begin{cases}1, & \text { if } x, y \in\left\{\frac{1}{(n+1)^{2}}: n \in \mathbb{N}\right\}, \\
0, & \text { otherwise. }\end{cases}
\end{aligned}
$$

It is clear that $p$ is a $w$-distance in $X$ and $(\psi, \phi) \in \Phi_{1} \times \Phi_{3}$. Let $x, y \in X$. In order to show that $f$ satisfies (6), we have to consider the following cases.

Case 1. $x, y \in\left\{\left(1 /(n+1)^{2}\right): n \in \mathbb{N}\right\}$. It follows that

$$
\begin{aligned}
\alpha(x, y) \int_{0}^{p(f x, f y)} \phi(t) \mathrm{d} t & =\int_{0}^{y^{4}} \mathrm{~d} t=y^{4} \leq \frac{1}{2} y^{2}=\psi\left(y^{2}\right) \\
& =\psi\left(\int_{0}^{p(x, y)} \phi(t) \mathrm{d} t\right) .
\end{aligned}
$$

Case 2. $x \notin\left\{\left(1 /(n+1)^{2}\right): n \in \mathbb{N}\right\} \quad$ or $\quad y \notin\left\{\left(1 /(n+1)^{2}\right)\right.$ : $n \in \mathbb{N}\}$. It is clear that

$$
\alpha(x, y) \int_{0}^{p(f x, f y)} \phi(t) \mathrm{d} t=0 \leq \psi\left(\int_{0}^{p(x, y)} \phi(t) \mathrm{d} t\right),
$$

that is, (6) holds. Let $x, y \in X$ with $\alpha(x, y) \geq 1$. It follows that $x, y \in\left\{\left(1 /(n+1)^{2}\right): n \in \mathbb{N}\right\}$ and

$$
\begin{aligned}
& f x=x^{2} \in\left\{\frac{1}{(n+1)^{2}}: n \in \mathbb{N}\right\}, \\
& f y=y^{2} \in\left\{\frac{1}{(n+1)^{2}}: n \in \mathbb{N}\right\},
\end{aligned}
$$

which yield that $\alpha(f x, f y)=1$. Hence, $f$ is an $\alpha$-admissible mapping. Put $x_{0}=(1 / 4) \in X$. It follows that $\alpha\left(x_{0}, f x_{0}\right)=$ $\alpha((1 / 4),(1 / 16))=1$. Finally, we have

$$
\frac{1}{(n+1)^{2}} \neq f \frac{1}{(n+1)^{2}}, \quad \forall n \in \mathbb{N},
$$

which implies that for each $y \in\left\{\left(1 /(n+1)^{2}\right): n \in \mathbb{N}\right\}$,

$$
\inf \{p(x, y)+p(x, f x): x \in X\}=y^{2}>0 \text {. }
$$

Hence, the conditions of Theorem 7 are satisfied. It follows from Theorem 7 that $f$ has a fixed point $0 \in X$. 


\section{Solvability of Nonlinear Integral Equations}

The theory of nonlinear integral equations nowadays is a large topic which is found in many applications of various branches in mathematics. In this section, we prove the existence results of solutions for the following nonlinear Fredholm and Volterra integral equations, respectively, by using Theorem 6:

$$
\begin{aligned}
& x(t)=\varphi(t)+\int_{a}^{b} K(t, s, x(s)) \mathrm{d} s, \quad \forall t \in[a, b], \\
& x(t)=\varphi(t)+\int_{a}^{t} K(t, s, x(s)) \mathrm{d} s, \quad \forall t \in[a, b],
\end{aligned}
$$

where $a, b \in \mathbb{R}$ are constants with $a<b, \varphi:[a, b] \longrightarrow \mathbb{R}$, and $K:[a, b]^{2} \times \mathbb{R} \longrightarrow \mathbb{R}$ are given functions.

Let $C([a, b], \mathbb{R})$ denote the Banach space of all continuous functions $x:[a, b] \longrightarrow \mathbb{R}$ with the norm $\|x\|=\sup _{t \in[a, b]}|x(t)|$. Put $X=C([a, b], \mathbb{R})$ and

$$
d(x, y)=\sup _{t \in[a, b]}|x(t)-y(t)|, \quad \forall x, y \in X .
$$

It is clear $(X, d)$ is a complete metric space. Define mappings $T$ and $S$ as follows:

$$
\begin{array}{r}
(T x)(t)=\varphi(t)+\int_{a}^{b} K(t, s, x(s)) \mathrm{d} s, \quad \forall(t, x) \in[a, b] \times X, \\
(S x)(t)=\varphi(t)+\int_{a}^{t} K(t, s, x(s)) \mathrm{d} s, \quad \forall(t, x) \in[a, b] \times X .
\end{array}
$$

Theorem 8. Let $\xi: \mathbb{R} \times \mathbb{R} \longrightarrow \mathbb{R}, \varphi:[a, b] \longrightarrow \mathbb{R}$, and $K:[a, b]^{2} \times \mathbb{R} \longrightarrow \mathbb{R}$ satisfy the following:

$\left(d_{1}\right) \varphi$ and $K$ are continuous $\left(d_{2}\right) \xi(T x(t), T y(t)) \geq 0$ and $\forall(t, x, y) \in[a, b] \times X^{2}$ with $\xi(x(t), y(t)) \geq 0$

$\left(d_{3}\right)$ there exists $x_{0} \in X$ such that $\xi\left(x_{0}(t), T x_{0}(t)\right) \geq 0$, $\forall t \in[a, b]$

$\left(d_{4}\right)$ if $\left\{x_{n}\right\}_{n \in \mathbb{N}}$ is a sequence in $X$ such that $x_{n} \longrightarrow x$ in $X$ and $\xi\left(x_{n}(t), x_{n+1}(t)\right) \geq 0, \forall(n, t) \in \mathbb{N} \times[a, b]$, then

$$
\xi\left(x_{n}(t), x(t)\right) \geq 0, \quad \forall(n, t) \in \mathbb{N} \times[a, b] .
$$

$\left(d_{5}\right)$ there exists $\psi \in \Phi_{1}$ with

$$
\begin{array}{r}
|K(t, s, y(s))| \leq \frac{\psi\left(\sup _{s \in[a, b]}|y(s)|\right)-|\varphi(t)|}{b-a}, \\
\forall(t, s, y) \in[a, b]^{2} \times X .
\end{array}
$$

Then, nonlinear Fredholm integral equation (48) has a solution in $X$.

Proof. Define $p: X \times X \longrightarrow \mathbb{R}^{+}, \phi: \mathbb{R}^{+} \longrightarrow \mathbb{R}^{+}$, and $\alpha: X \times$ $X \longrightarrow \mathbb{R}^{+}$by

$$
\begin{gathered}
p(x, y)=\sup _{t \in[a, b]}|y(t)|, \quad \forall x, y \in X, \\
\phi(r)=1, \quad \forall r \in \mathbb{R}^{+},
\end{gathered}
$$

$\alpha(x, y)=\left\{\begin{array}{ll}1 & \text { if } \xi(x(t), y(t)) \geq 0, \forall t \in[a, b] \\ 0 & \text { otherwise }\end{array}, \quad \forall x, y \in X\right.$

Clearly, $p$ is a $w$-distance in $X$ and $\left(c_{1}\right),\left(c_{2}\right)$, and $\left(c_{4}\right)$ follow from $\left(d_{2}\right),\left(d_{3}\right)$, and $\left(d_{4}\right)$, respectively. It is easy to verify that $\left(d_{1}\right)$ and (51) ensure that, for each $x \in X, T x$ is a continuous function in $[a, b]$, which yields that $T$ maps $X$ into itself. Observe that $(51)$ and $\left(d_{5}\right)$ mean that

$$
\begin{aligned}
\int_{0}^{|(T y)(t)|} \phi(r) \mathrm{d} r=|(T y)(t)| & =\left|\varphi(t)+\int_{a}^{b} K(t, s, y(s)) \mathrm{d} s\right| \\
& \leq|\varphi(t)|+\int_{a}^{b}|K(t, s, y(s))| \mathrm{d} s \\
& \leq|\varphi(t)|+\int_{a}^{b} \frac{\psi\left(\sup _{s \in[a, b]}|y(s)|\right)-|\varphi(t)|}{b-a} \mathrm{~d} s \\
& =|\varphi(t)|+\frac{1}{b-a} \int_{a}^{b}[\psi(p(x, y))-|\varphi(t)|] \mathrm{d} s \\
& =\psi\left(\int_{0}^{p(x, y)} \phi(r) \mathrm{d} r\right), \quad \forall(t, x, y) \in[a, b] \times X^{2},
\end{aligned}
$$

which implies that 


$$
\begin{aligned}
\alpha(x, y) \int_{0}^{p(T x, T y)} \phi(r) \mathrm{d} r & =\alpha(x, y) \int_{0}^{\sup _{t \in[a, b]}|(T y)(t)|} \phi(r) \mathrm{d} r \\
& \leq \psi\left(\int_{0}^{p(x, y)} \phi(r) \mathrm{d} r\right), \quad \forall x, y \in X .
\end{aligned}
$$

That is, (6) holds. Thus, Theorem 6 guarantees that $T$ has a fixed point $x \in X$, which is a solution of nonlinear Fredholm integral equation (48) in $X$. This completes the proof.

As in the proof of Theorem 8, we get the following result and omit its proof.

Theorem 9. Let $\xi: \mathbb{R} \times \mathbb{R} \longrightarrow \mathbb{R}, \varphi:[a, b] \longrightarrow \mathbb{R}$, and $K:[a, b]^{2} \times \mathbb{R} \longrightarrow \mathbb{R}$ satisfy $\left(d_{1}\right),\left(d_{4}\right),\left(d_{5}\right)$, and

$\left(d_{6}\right) \xi(S x(t), S y(t)) \geq 0, \forall(t, x, y) \in[a, b] \times X^{2}$ with $\xi(x(t), y(t)) \geq 0$;

$\left(d_{7}\right)$ there exists $x_{0} \in X$ such that $\xi\left(x_{0}(t), S x_{0}(t)\right) \geq 0$, $\forall t \in[a, b]$. Then, nonlinear Volterra integral equation (49) has a solution in X.

\section{Data Availability}

The data used to support the findings of this study are included within the article.

\section{Conflicts of Interest}

The authors declare that they have no conflicts of interest.

\section{Authors' Contributions}

All authors contributed equally to the writing of this paper. All authors read and approved the final manuscript.

\section{Acknowledgments}

This work was supported by the National Natural Science Foundation of China (no. 41701616).

\section{References}

[1] A. Branciari, "A fixed point theorem for mappings satisfying a general contractive condition of integral type," International Journal of Mathematics and Mathematical Sciences, vol. 29, no. 9, pp. 531-536, 2002.

[2] B. Samet, C. Vetro, and P. Vetro, "Fixed point theorems for $\alpha-\psi$-contractive type mappings," Nonlinear Analysis: Theory, Methods \& Applications, vol. 75, no. 4, pp. 2154-2165, 2012.

[3] O. Kada, T. Suzuki, and W. Takahashi, "Nonconvex minimization theorems and fixed point theorems in complete metric spaces," Mathematica Japonicae, vol. 44, pp. 381-391, 1996.

[4] M. Imdad and F. Rouzkard, "Fixed point theorems in ordered metric spaces via $w$-distances," Fixed Point Theory and Applications, vol. 2012, no. 1, 17 pages, 2012.

[5] F. Rouzkard, M. Imdad, and D. Gopal, "Some existence and uniqueness theorems on ordered metric spaces via generalized distances," Fixed Point Theory and Applications, vol. 2013, no. 1, 45 pages, 2013.

[6] Y. Sang and Q. Meng, "Fixed point theorems with generalized altering distance functions in partially ordered metric spaces via $w$-distance and applications," Fixed Point Theory and Applications, vol. 2015, no. 1, 25 pages, 2015.

[7] R. Zuhra, M. S. M. Noorani, and F. Shaddad, "Contraction mapping principle in partially ordered quasi metric space concerning to $w$-distances," The Journal of Nonlinear Sciences and Applications, vol. 10, no. 2, pp. 699-712, 2017.

[8] H. Lakzian, D. Gopal, and W. Sintunavarat, "New fixed point results for mappings of contractive type with an application to nonlinear fractional differential equations," Journal of Fixed Point Theory and Applications, vol. 18, no. 2, pp. 251-266, 2016.

[9] Z. Liu, X. Li, S. M. Kang, and S. Y. Cho, "Fixed point theorems for mappings satisfying contractive conditions of integral type and applications," Fixed Point Theory and Applications, vol. 2011, no. 1, 64 pages, 2011.

[10] M. Marcelina and P. Valeriu, "Some fixed point theorems for mappings satisfying implicit relations in symmetric spaces," Libertas Mathematica, vol. 28, pp. 1-13, 2008. 